\title{
Regional anesthesia for an upper extremity amputation for palliative care in a patient with end-stage osteosarcoma complicated by a large anterior mediastinal mass
}

\author{
This article was published in the following Dove Press journal: \\ Journal of Pain Research \\ 24 September 2015 \\ Number of times this article has been viewed
}

\author{
Mumin Hakim' \\ Candice Burrier' \\ Tarun Bhalla' \\ Vidya T Raman' \\ David P Martin ${ }^{1,2}$ \\ Olamide Dairo' \\ Joel L Mayerson ${ }^{3,4}$ \\ Joseph D Tobias ${ }^{1,2}$ \\ 'Department of Anesthesiology \\ and Pain Medicine, Nationwide \\ Children's Hospital, ${ }^{2}$ Department of \\ Anesthesiology and Pain Medicine, \\ The Ohio State University, \\ ${ }^{3}$ Department of Orthopedic Surgery, \\ Nationwide Children's Hospital, \\ ${ }^{4}$ Department of Orthopedic Surgery, \\ The Ohio State University, Columbus, \\ OH, USA
}

Correspondence: Tarun Bhalla Department of Anesthesiology and Pain Medicine, Nationwide Children's Hospital, 700 Children's Drive,

Columbus, OH 43205, USA

Tel +l 6147224200

Fax + I 6I4 7224203

Email tarun.bhalla@nationwidechildrens.org

\begin{abstract}
Tumor progression during end-of-life care can lead to significant pain, which at times may be refractory to routine analgesic techniques. Although regional anesthesia is commonly used for postoperative pain care, there is limited experience with its use during home hospice care. We present a 24-year-old male with end-stage metastatic osteosarcoma who required anesthetic care for a right-sided above-the-elbow amputation. The anesthetic management was complicated by the presence of a large mediastinal mass, limited pulmonary reserve, and severe chronic pain with a high preoperative opioid requirement. Intraoperative anesthesia and postoperative pain management were provided by regional anesthesia using an interscalene catheter. He was discharged home with the interscalene catheter in place with a continuous local anesthetic infusion that allowed weaning of his chronic opioid medications and the provision of effective pain control. The perioperative applications of regional anesthesia in palliative and home hospice care are discussed.
\end{abstract}

Keywords: osteosarcoma, peripheral nerve block, palliative care

\section{Introduction}

Osteosarcoma is the most common malignant bone tumor in children and young adults, having a peak incidence in the second decade and the sixth decade or later, thereby representing a bimodal distribution. ${ }^{1-3}$ Approximately 800 cases of osteosarcomas are diagnosed in the US every year. Metaphyseal regions of the distal femur, proximal tibia, and proximal humerus are frequent sites of origin. Approximately $90 \%$ of the metastasis occurs in the lungs, and $10 \%-15 \%$ of patients have detectable metastases at the time of diagnosis. Today, $90 \%-95 \%$ of patients with osteosarcoma can be treated with limb-sparing surgery, and $60 \%-80 \%$ of patients with localized disease are longterm survivors. Despite the improvements in care, disease progression and metastatic disease can lead to debilitating consequences and death. During the terminal stages of the disease, local and metastatic disease may result in significant pain that is refractory to conventional oral and intravenous opioids.

We present a 24-year-old male with end-stage metastatic osteosarcoma who required anesthetic care for a right-sided above-the-elbow amputation. The anesthetic management was complicated by the presence of a large mediastinal mass, limited pulmonary reserve, and severe chronic pain with a high preoperative opioid requirement. Intraoperative surgical anesthesia and postoperative pain management were provided by regional anesthesia using an interscalene catheter. The perioperative 
applications of regional anesthesia in palliative and home hospice care are discussed.

\section{Case}

Institutional Review Board approval is not required at Nationwide Children's Hospital (Columbus, OH, USA) for the presentation of single case report. The patient was a 24 -yearold, $79 \mathrm{~kg}$ male who presented for a palliative right-sided, above-the-elbow amputation for recurrent osteosarcoma of the right arm with intractable pain. His medical history was significant for osteosarcoma of the right distal radius with metastatic disease to the lungs, status after two cycles of palliative chemotherapy that included methotrexate, doxorubicin, and cisplatin. He previously had undergone a right below-theelbow amputation. At the time of presentation, he had electively discontinued his palliative chemotherapy. He presented with local progression of the tumor into the humerus and axilla as well as progression of the lung metastases with severe pain in the affected extremity. The right arm mass measured $15 \mathrm{~cm} \times 14 \mathrm{~cm} \times 18 \mathrm{~cm}$ (Figure 1). He was being followed by the palliative care service and requested surgical options for treatment of his pain. Computed tomography demonstrated an $8.1 \mathrm{~cm} \times 6.8 \mathrm{~cm} \times 6.4 \mathrm{~cm}$ anterior mediastinal mass at the level of the transverse aortic arch with severely narrowed left innominate vein with dilated collateral vessels and displaced left pulmonary artery (Figure 2). He also had a left inferior posterior pulmonary mass, multiple pulmonary nodules, and a left lower lobe infiltrate. Current home medications included self-prescribed high-dose vitamin C. The current pain medication schedule had escalated to include hydromorphone $4 \mathrm{mg}$ orally (PO) every 3 hours as needed, methadone $10 \mathrm{mg}$ PO every 6 hours, morphine $15 \mathrm{mg}$ PO every 3 hours as needed, and gabapentin $300 \mathrm{mg}$ PO four times per day. Although the patient was generally noncompliant with opioid medications

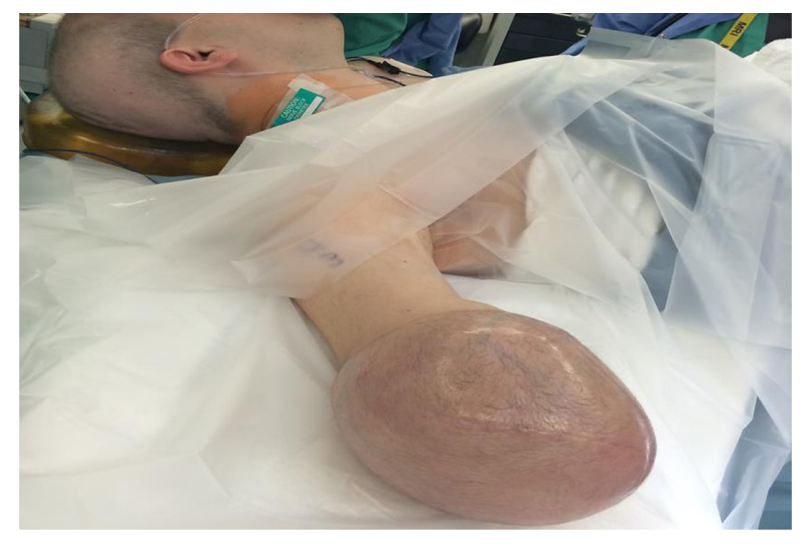

Figure I Preoperative photograph showing tumor progression and involvement of the area of previous amputation.

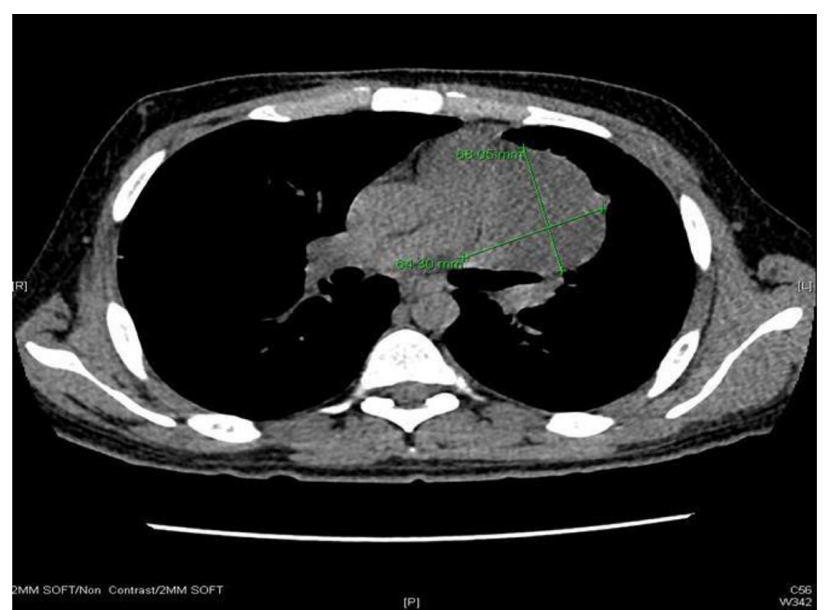

Figure 2 Preoperative computed tomography scan demonstrating a large anterior mediastinal mass.

and not taking them as prescribed, his daily oral morphine equivalents were equal to $568 \mathrm{mg}$. He had no known drug or environmental allergies.

Preoperative physical examination revealed a young man with a large mass at the site of the previous right below-theelbow amputation extending to the proximal humerus. He was in moderate distress rating the pain in his arm as $7 / 10$. He was able to lie flat without shortness of breath with an oxygen saturation of $99 \%$. Airway examination was normal. Preoperative laboratory evaluation including electrolytes, renal function, coagulation function, blood glucose, and hepatic function was within normal limits. The hemoglobin was $14.1 \mathrm{~g} / \mathrm{dL}$ with a hematocrit level of $42.6 \%$. The anesthesia team had a detailed discussion of anesthetic options with the patient as well as risks and benefits of anesthesia. Risks of prolonged endotracheal intubation and further cardiopulmonary compromise due to the large anterior mediastinal mass were discussed. It was also made clear that there was a risk of intraoperative death. It was ultimately decided that the safest course was a regional anesthetic technique. The patient was transported to the operating room, and routine American Society of Anesthesiologists' monitors were applied. A nasal cannula with $2 \mathrm{~L}$ of oxygen was administered, and the patient was positioned supine. The patient was then prepped and draped in sterile fashion for placement of an interscalene catheter. A high supraclavicular/low interscalene approach was used, and a catheter was

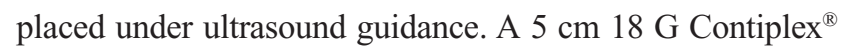
needle was used with a $20 \mathrm{G}$ catheter (B. Braun, Bethlehem, PA, USA). A test dose of $2 \mathrm{~mL}$ of $0.5 \%$ ropivacaine with 1:200,000 epinephrine was administered and was negative. A total of $15 \mathrm{~mL}$ of $0.5 \%$ ropivacaine with 1:200,000 epinephrine and $3 \mathrm{mg}$ dexamethasone was administered through 
the needle. The catheter was then advanced into position, and placement confirmed by ultrasound. The catheter was secured with Dermabond ${ }^{\circledR}$ topical skin adhesive (Ethicon, Johnson and Johnson, New Brunswick, NJ, USA), Steri-strips ${ }^{\mathrm{TM}}$, and a clear occlusive dressing. An intercostobrachial nerve block was performed using anatomical landmarks with a total of $13 \mathrm{~mL}$ of $0.5 \%$ ropivacaine with 1:200,000 epinephrine. After 20 minutes, surgical anesthesia was present without any change in the patient's respiratory status. Sedation was achieved with incremental doses of midazolam, dexmedetomidine, and ketamine. The estimated blood loss was $250 \mathrm{~mL}$. Intraoperative fluids included 1,000 mL of Lactated Ringer's solution. Following completion of the surgical procedure, the patient was transferred to the post-anesthesia care unit. An elastomeric pump was attached to the low interscalene nerve block catheter with an infusion of $0.2 \%$ ropivacaine at $6 \mathrm{~mL} / \mathrm{h}$. The patient was transferred to the inpatient unit for observation overnight. The patient rated his pain overnight as a $0 / 10$. He refused his chronic pain medications overnight. On postoperative day one, the patient was seen sitting up in bed in no apparent distress. He stated that his pain in his arm was nonexistent. He stated that it just "felt weird" from the nerve block. He was interested in keeping the nerve block catheter for a prolonged amount of time, and therefore, it was coordinated with his palliative home health nurse to deliver and change the local anesthetic bags for the nerve block catheter pump. He was discharged on postoperative day one. The patient was followed by daily phone calls from the acute pain service team. The patient was given explicit written instructions of care of the peripheral nerve catheter and a phone number to contact in case of questions. The palliative care home nurse traveled to his home every 4 days to change the local anesthetic bag and to inspect the catheter site and dressing. During this time, he had minimal pain and was able to completely wean off of his high doses of preoperative opioid. The patient kept the nerve block catheter for a total of 8 days, after which he pulled the catheter out himself at home. After discontinuation of the nerve block catheter, the patient continued to have low levels of pain and needed minimal opioids for his pain. The patient was very satisfied with his experience with the home peripheral nerve catheter. Per his wishes, he expired comfortably 3 months later at home with wife.

\section{Comments}

Regional anesthetic techniques are commonly used to provide intraoperative surgical anesthesia as well as postoperative analgesia. In addition to their applications during the perioperative period, they may be useful to control acute and chronic pain of various other etiologies. Our patient presented with a high-grade metastatic osteosarcoma with severe pain related to local tumor progression and cardiorespiratory compromise related to metastatic involvement of the lungs and an anterior mediastinal mass. ${ }^{4}$ Given his limited pulmonary reserve and the presence of an anterior mediastinal mass, it was decided to proceed with a regional anesthetic technique to provide surgical anesthesia as well as postoperative analgesia. To date, there are a limited number of case reports outlining the use of regional anesthesia as the primary anesthetic technique for upper extremity amputations..$^{5-7}$

Various options exist to provide brachial plexus anesthesia. ${ }^{8,9}$ In our patient, surgical anesthesia of the proximal humerus coverage was required which limited the regional anesthetic options to an interscalene or a supraclavicular block. Despite its advantages as a means of avoiding the need for general anesthesia, brachial plexus anesthesia may compromise respiratory function due to phrenic nerve blockade and hemidiaphragmatic paralysis (HDP), especially in the presence of comorbid disease. HDP is a potential complication of both interscalene and supraclavicular blocks, leading to a $30 \%$ decline in pulmonary function which may not be tolerated in a patient with limited pulmonary reserve. It has been reported that HDP occurs in $100 \%$ of patients following interscalene blockade, whereas the incidence varies significantly (1\%-75\%) after supraclavicular block has been reported. ${ }^{10-16}$ More recently, the use of ultrasound has been shown to decrease the incidence of phrenic nerve blockade during the supraclavicular approach to the brachial plexus. ${ }^{17}$ In our patient, a low interscalene/ high supraclavicular approach using ultrasound guidance was chosen to potentially limit the risk of HDP and yet provide surgical anesthesia of the proximal humerus. An intercostobrachial nerve block was performed to provide anesthesia for the tourniquet during the procedure. If there are concerns regarding HDP, it is feasible to evaluate diaphragmatic motion using ultrasound after the block is placed while the patient is in the operating room.

The latest advent in regional anesthesia has been the use of outpatient continuous peripheral nerve block (CPNB) catheters with elastomeric or digital infusion devices to provide ongoing analgesia following major outpatient surgical procedures. ${ }^{18,19}$ Although these catheters for postoperative pain are generally left in situ for only a few days, there are reports of their extended use for palliative pain control. ${ }^{19-23}$ The main concern with long-term indwelling catheters is the risk of serious local and deep tissue infections. Anghelescu et al reviewed their experience with 179 CPNB catheters in 116 pediatric hematology/oncology patients (mean age 
of 15.1 years). ${ }^{24}$ There were only two $(1.12 \%)$ infectious complications noted in their cohort, both associated with femoral catheters. The infections were mild and necessitated catheter removal at days 10 and 13 . Between $23 \%$ and 57\% of CPNB catheters become colonized with bacteria, but only $0 \%-3 \%$ result in localized infections, and $<1 \%$ result in serious infections. ${ }^{25}$ The low incidence and severity of infections in various studies indicate that CPNB catheters are a safe method of pain control that carries a limited infection risk, even in the setting of prolonged catheter use and in a potentially immunocompromised patient population. Subcutaneous tunneling of the catheter has been suggested as another option for prolonged use of such catheters to further minimize the risk of infection. ${ }^{26}$ Additionally, as with central venous access, the use of a chlorhexidine-impregnated dressing has been suggested as a means of decreasing the incidence of infectious complications of indwelling regional anesthesia catheters. ${ }^{27}$

In conclusion, the use of regional anesthesia in our patient avoided the potential complications of general anesthesia in patient with metastatic lung disease and a mediastinal mass. This allowed for successful surgical resection and a reduction in the pain caused by the expanding tumor mass. Use of the catheter at home provided effective analgesia and eliminated the need for high-dose opioids. The reduction in pain allowed him to resume his daily life activities. Although there has been a significant increase in the use of regional anesthesia during the perioperative period, our anecdotal success suggests that such techniques may also have applications in the treatment of pain during end-of-life care. ${ }^{28}$

\section{Disclosure}

The authors report no conflicts of interest in this work.

\section{References}

1. Vander Griend RA. Osteosarcoma and its variants. Med Clin North Am. 1996;27:575-581.

2. Mirabello L, Troisi RJ, Savage SA. Osteosarcoma incidence and survival rates from 1973 to 2004: data from the surveillance, epidemiology, and end results program. Cancer. 2009;115:1531-1543.

3. Glasser DB, Lane JM, Huvos AG, Marcove RC, Rosen G. Survival, prognosis, and therapeutic response in osteogenic sarcoma. The Memorial Hospital experience. Cancer. 1992;69:698-708.

4. Pearson JK, Tan GM. Pediatric anterior mediastinal mass: a review article. Semin Cardiothorac Vasc Anesth. 2015;19(3):248-254.

5. Meola S, Olivieri M, Mirabile C, Mastrandrea P. Anesthetic management for right upper extremity amputation due to recidivous cutaneous carcinoma and acute postoperative pain control in patients affected by epidermolysis bullosa. Minerva Anestesiol. 2010;76:144-147.

6. de Moraes JA, Reis TJ, Naspolini Filho H, Batista TP, de Lucena MA. Forequarter amputation under locoregional anesthesia. Rev Col Bras Cir. 2013;40:427-429.

7. Kuş A, Gürkan Y, Gök CN, Solak M, Toker K. Infraclavicular block with ultrasound at amputated upper extremity. Agri. 2010;22: 134-136.
8. Tobias JD. Brachial plexus anesthesia in children. Paediatr Anaesth. 2001;11:265-275.

9. Neal JM, Gerancher JC, Hebl JR, et al. Upper extremity regional anesthesia: essentials of our current understanding. Reg Anesth Pain Med. 2009;34:134-170.

10. Urmey WF, Talts KH, Sharrock NE. One hundred percent incidence of hemidiaphragmatic paresis associated with interscalene brachial plexus anesthesia as diagnosed by ultrasonography. Anesth Analg. 1991;72:498-503.

11. Urmey WF, McDonald M. Hemidiaphragmatic paresis during interscalene brachial plexus block: effects on pulmonary function and chest wall mechanics. Anesth Analg. 1992;74:352-357.

12. Pere P, Pitkänen M, Rosenberg PH, et al. Elect of continuous interscalene brachial plexus block on diaphragm motion and ventilatory function. Acta Anaesthesiol Stand. 1992;36:53-57.

13. Dhuner KG, Mober E, Onne L. Paresis of the phrenic nerve during brachial plexus block analgesia and its importance. Acta Chir Stand. 1955;109:53-57.

14. Knoblanche GE. The incidence and aetiology of phrenic nerve blockade associated with supraclavicular brachial plexus block. Anaesth Intensive Care. 1979;7:346-349.

15. Shaw WM. Paralysis of the phrenic nerve during brachial plexus anesthesia. Anesthesiology. 1949;10:627-628.

16. Neal JM, Moore JM, Kopacz DJ, Liu SS, Kramer DJ, Plorde JJ. Quantitative analysis of respiratory, motor, and sensory function after supraclavicular block. Anesth Analg. 1998;86:1239-1244.

17. Renes SH, Spoormans HH, Gielen MJ, Rettig HC, van Geffen GJ. Hemidiaphragmatic paresis can be avoided in ultrasound-guided supraclavicular brachial plexus block. Reg Anesth Pain Med. 2009;34:595-599.

18. Chelly JE, Ghisi D, Fanelli A. Continuous peripheral nerve blocks in acute pain management. Br J Anaesth. 2010;105:86-96.

19. Kaddoum RN, Burgoyne LL, Pereiras JA, Germain M, Neel M, Anghelescu DL. Nerve sheath catheter analgesia for forequarter amputation in paediatric oncology patients. Anaesth Intensive Care. 2013;41:671-677.

20. Dadure C, Bringuier S, Raux O, et al. Continuous peripheral nerve blocks for postoperative analgesia in children: feasibility and side effects in a cohort study of 339 catheters/Blocs nerveux périphériques continus pour 1'analgésie postopératoire chez l'enfant: efficacité et effets secondaires dans une cohorte de 339 cathéters. Can J Anaesth. 2009;56:843-850.

21. Ganesh A, Rose JB, Wells L, et al. Continuous peripheral nerve blockade for inpatient and outpatient postoperative analgesia in children. Anesth Analg. 2007;105:1234-1242.

22. Capdevila $X$, Bringuier S, Borgeat A. Infectious risk of continuous peripheral nerve blocks. Anesthesiology. 2009;110:182-188.

23. Capdevila X, Pirat P, Bringuier S, et al; French Study Group on Continuous Peripheral Nerve Blocks. Continuous peripheral nerve blocks in hospital wards after orthopedic surgery: a multicenter prospective analysis of the quality of postoperative analgesia and complications in 1,416 patients. Anesthesiology. 2005;103:1035-1045.

24. Anghelescu DL, Harris BL, Faughnan LG, et al. Risk of catheter-associated infection in young hematology/oncology patients receiving long-term peripheral nerve blocks. Paediatr Anaesth. 2012;22:1110-1116.

25. Cuvillon P, Ripart J, Lalourcey L, et al. The continuous femoral nerve block catheter for postoperative analgesia: bacterial colonization, infectious rate and adverse effects. Anesth Analg. 2001;93:1045-1049.

26. Pacenta HL, Kaddoum RN, Pereiras LA, Chidiac EJ, Burgoyne LL. Continuous tunnelled femoral nerve block for palliative care of a patient with metastatic osteosarcoma. Anaesth Intensive Care. 2010;38:563-565.

27. Kerwat K, Eberhart L, Kerwat M, et al. Chlorhexidine gluconate dressings reduce bacterial colonization rates in epidural and peripheral regional catheters. Biomed Res Int. 2015;2015:149785.

28. Anghelescu DL, Faughnan LG, Baker JN, Yang J, Kane JR. Use of epidural and peripheral nerve blocks at the end of life in children and young adults with cancer: the collaboration between a pain service and a palliative care service. Pediatr Anesth. 2010;20:1070-1077. 
Journal of Pain Research

\section{Publish your work in this journal}

The Journal of Pain Research is an international, peer-reviewed, open access, online journal that welcomes laboratory and clinical findings in the fields of pain research and the prevention and management of pain. Original research, reviews, symposium reports, hypothesis formation and commentaries are all considered for publication.

The manuscript management system is completely online and includes a very quick and fair peer-review system, which is all easy to use. Visit http://www.dovepress.com/testimonials.php to read real quotes from published authors.

\footnotetext{
Submit your manuscript here: http://www.dovepress.com/journal-of-pain-research-journal
} 